\title{
ZEITSCHRIF'T
}

FŬR

\section{ÄGYPTISCHE SPRACHE DND}

\section{ALTERTHUMSKUNDE}

\author{
HERAUSGEGEBEN \\ voN \\ C. R. LEPSIUS \\ ZU BERLIN
}

UNTER MITWIRKUNG VON H. BRUGSCH ZU GÖTTINGEN

\section{SE CHSTER JAHR GA N G \\ 1868}

LEIPZIG

J. C. HINRICHS'SCHE B U CHHANDLUNG 
